\title{
Breathing circuit disconnections: averting catastrophes
}

\author{
Steven Dain MD FRCPC
}

$\mathrm{T}$

HE case reported by Kennedy and French in this issue of the Canadian Journal of Anesthesia, illustrates several important points in the practice of anesthesiology. ${ }^{1}$ These include, but are not limited to, the type of monitors used, the variables that are measured, the appropriate use of alarm settings and the use and reuse of single use and reusable equipment.

Circuit disconnections are not uncommon. They result from patient movement, surgical manipulation of the patient, gravity, through the weight of the circuit and the inherent "springiness" of the circuit pulling the "friction-fit" connections apart. Several factors contribute to breathing circuit disconnections. The large number of connections between the laryngeal mask airway (LMA) or endotracheal tube, the heat and moisture exchanger (HME), the airway gas sampling port, the side stream spirometer sampling port and the breathing circuit increase the probability of disconnections. The precision of fit, material, age and amount of wear influence the quality of the connection.

Reusable connectors, tubing and sampling port adapters wear out and crack over time. In addition, reusable equipment may not have been reprocessed according to the manufacturers' instructions, which may cause physical changes in the connectors' dimensions. The very small manufacturing tolerances of our standard $15 \mathrm{~mm}$ and $22 \mathrm{~mm}$ connectors may be violated after several use/cleaning cycles. ${ }^{2}$ This is more likely a problem for plastic single use items that are not specifically manufactured to withstand the rigours of washing and re-sterilization and have been reprocessed against the advice of the manufacturer. This is one of the risks that must be evaluated and accepted when organizations reprocess and reuse items intended for single use only. All equipment must be adequately inspected prior to use. Taping the connections together from the LMA to the breathing circuit Y-piece reduces the risk of disconnection.
The authors noted that they detected the disconnection through a decrease in the end-tidal anesthetic levels, not by the changes in tidal volume as their minute volume alarm was turned off (manufacturer default). The use of a HME with an integrated gas sampling port is advantageous as it decreases the number of connections between the LMA and the breathing circuit. However, the changes in the measured airway gas content (inspired and expired anesthetic agent, $\mathrm{CO}_{2}, \mathrm{O}_{2}$ ) and spirometry values will vary depending on the location of the disconnection. It would be an interesting exercise to predict the changes to expect from each combination and order of monitoring ports and each possible point of disconnection.

In this case report, a Datex-Ohmeda AS/3 monitoring system with side-stream spirometry was used. Instead of monitoring trends, this monitor with the side stream spirometry module, is capable of continuously displaying a flow-volume or pressure-volume loop in its "split screen" mode. The use of the split screen flow-volume loops for spontaneously breathing patients would aid in the detection of changes in the lung, chest wall and in the breathing circuit and would provide another means of identification of patient circuit disconnection.

The authors suggest that in the future, monitoring equipment should have "smart alarm systems". Although intelligent alarm systems may be highly desirable, they will increase the cost of the monitoring equipment, as the algorithms must be validated by clinical trials prior to regulatory agency approval. Current monitors without intelligent alarm systems, do not require clinical trials. Even if manufacturers could design the "best alarm system in the world", it is up to the operators of the equipment to use those alarm systems appropriately. The best alarm systems will not function if they are disabled or the alarm limits are set to extreme values. The authors stated that the minute volume alarm was turned off, which is the manufacturer default on the AS $/ 3$ monitor. ${ }^{3}$ 
Anesthesia departments and equipment users must examine the manufacturer defaults of all parameters of all equipment in use and decide whether to accept them or not. Most modern microprocessor based equipment allows for storage of one or more sets of default alarm limit settings ("USER defined limits") that may be optimized for their own patient population and environment of use. In addition, some equipment allows individuals to store their own set of alarm limits ("OPERATOR defined limits").

The International Electrotechnical Commission (IEC) and the Organization for International Standardization (ISO) currently have a Joint Working Group on Alarm Systems for Medical Electrical Equipment that will set new standards for the design and implementation of alarm systems and provide guidance to manufacturers in the implementation of alarm systems for all medical equipment. ${ }^{4,5}$ In order to improve equipment functionality, standards for usability are available ${ }^{6}$ and in the future regulators will require manufacturers to perform usability testing.?

All the connectors that we use should be compliant with ISO 5356-1:1996; Anaesthetic and respiratory equipment - Conical connectors - Part 1: Cones and sockets and the breathing circuits with ISO 5367:2000; Breathing tubes intended for use with anesthetic apparatus and ventilators. These standards provide the dimensions, functional requirements and methods for testing the force required to separate 15 $\mathrm{mm}$ and $22 \mathrm{~mm}$ male and female connectors. The standards state "breathing tubes shall not become detached from the appropriate male conical connector with a force less than 40 Newtons" 8 (about a $4 \mathrm{~kg}$ weight hanging from the tubing). The Y-piece shall not become detached from the breathing tube with a force of less than 45 Newtons. ${ }^{9}$ These specifications must be maintained after cleaning.

Purchasing quality monitors, breathing circuits and connectors, appropriate cleaning of reusable items, ${ }^{10}$ vigilance in inspecting the equipment for wear prior to use, using monitor technology to its fullest extent using appropriate alarm settings, and vigilance with situational awareness during the administration of anesthesia all contribute to the best possible patient outcomes.

\section{Les déconnexions du circuit respiratoire : éviter le pire}

Le cas cité par Kennedy et French dans le présent numéro du Journal canadien d'anesthésie illustre certains aspects importants de l'exercice de l'anesthésie. ${ }^{1}$ Ils mentionnent, entre autres, le type de moniteurs utilisés, les variables mesurées, le réglage approprié des avertisseurs et l'utilisation et la réutilisation de matériel jetable et réutilisable.

Les déconnexions de circuit ne sont pas rares. Elles sont causées par un mouvement du patient, la manipulation chirurgicale du patient, la gravité, le poids du circuit et son "élasticité" inhérente qui tend à rompre "l'ajustement serré" des connexions. Certains facteurs contribuent aussi aux déconnexions. En effet, le nombre de connexions entre le masque laryngé (ML) ou le tube endotrachéal, l'échangeur de chaleur et d'humidité $(\mathrm{ECH})$, l'accès au prélèvement de gaz expirés, le site de prélèvement du spiromètre à soutirage latéral et le circuit respiratoire augmente les possibilités de déconnexions. La précision de l'ajustement, le matériau, l'âge et le degré d'usure influencent la qualité de la connexion.

Les connecteurs réutilisables, les adaptateurs fixés aux sites de prélèvement et aux tubulures s'usent et se fissurent avec le temps. De plus, il est possible que le matériel réutilisable n'ait pas été récupéré selon les directives des manufacturiers, ce qui peut modifier les dimensions physiques des connecteurs. Les très faibles tolérances de fabrication de nos connecteurs standard de 15 et de $22 \mathrm{~mm}$ ne seront peut-être plus les mêmes après quelques cycles d'utilisation et de nettoyage. ${ }^{2}$ C'est plus susceptible de se produire avec les articles jetables en plastique, non spécifiquement fabriqués pour résister à la rigueur du lavage et de la restérilisation et récupérés malgré l'avis contraire du fabricant. Les organismes qui récupèrent et réutilisent des articles supposés jetables doivent donc évaluer et accepter ce risque. Par ailleurs, une inspection adéquate avant usage et la fixation des connexions avec du ruban adhésif, du ML à la pièce en $\mathrm{Y}$ du circuit respiratoire, réduisent le risque de déconnexion.

Les auteurs rapportent avoir détecté une déconnexion par la baisse des niveaux d'anesthésique de fin d'expiration, non par les changements de volume courant, l'alarme de la ventilation minute étant en 
position d'arrêt (disposition implicite choisie par le fabricant). L'avantage d'un ECH muni d'un site de prélèvement intégré est le nombre réduit de connexions entre le ML et le circuit respiratoire. Cela dit, les modifications du contenu des gaz respirés (anesthésiques inspirés et expirés, le $\mathrm{CO}_{2}, \mathrm{l}^{\prime} \mathrm{O}_{2}$ ) et les valeurs spirométriques varient selon la localisation de la déconnexion. Il serait intéressant de prédire les changements possibles pour toute combinaison ou tout agencement des sites de monitorage et pour tout point de déconnexion.

Au cours de la présente observation, un système de monitorage Datex-Ohmeda AS $/ 3$, muni d'un spiromètre à soutirage latéral, a été utilisé. Au lieu d'indiquer les variations, ce moniteur peut afficher continûment sur son "écran divisé" une boucle débitvolume ou pression-volume. L'utilisation, pour les patients qui respirent spontanément, de boucles débitvolume sur écran divisé peut favoriser la détection de changements pulmonaires, des changements dans la paroi thoracique ou dans le circuit respiratoire et fournir d'autres moyens de déceler une déconnexion dans le circuit du patient.

Les auteurs recommandent qu'à l'avenir le matériel de surveillance soit muni "d'avertisseurs intelligents". Quoique hautement souhaitables, ces avertisseurs augmentent le coût du matériel de surveillance, car les algorithmes doivent être validés par des essais cliniques antérieurs à l'homologation par un organisme de réglementation. Ce qui n'est pas le cas des moniteurs courants, sans avertisseurs intelligents. Même si on pouvait mettre au point les "meilleurs avertisseurs au monde", l'usager devrait toujours s'en servir convenablement. Les meilleurs avertisseurs ne fonctionnent pas s'ils sont hors service ou si les seuils d'alerte sont réglés selon des valeurs extrêmes. Les auteurs ont déclaré que l'alarme de ventilation minute était en position d'arrêt, ce qui est la position implicite choisie par le fabricant sur le moniteur AS $/ 3$. $^{3}$

Les usagers de services et de matériel d'anesthésie doivent vérifier les positions implicites choisies par le fabricant pour tous les paramètres de tout matériel utilisé et décider s'ils les gardent ou pas. La majorité du matériel microprocessé permet la mise en mémoire d'un ensemble, ou plus, de réglages implicites des seuils d'alerte de l'avertisseur ("Seuils configurés par l'UTILISATEUR") qui peuvent être optimalisés pour leur propre population de patients et les conditions d'utilisation. En outre, avec certains équipements il est possible de mémoriser son propre ensemble de seuils d'alerte ("Seuils configurés par l'OPÉRATEUR")

Un groupe de travail mixte, issu de La Commission électrotechnique internationale (CEI) et de
l'Organisation internationale de normalisation (ISO), étudie les avertisseurs pour équipement électrique médical afin de définir de nouvelles normes de conception et de mise en application des avertisseurs et de fournir des indications d'installation aux fabricants d'équipement médical. ${ }^{4,5}$ Dans le but d'améliorer la fonctionnalité de l'équipement, on peut consulter les normes pour la facilité d'utilisation ${ }^{6}$. D'ailleurs, à l'avenir, les organismes de réglementation vont exiger des fabricants qu'ils réalisent des tests d'utilisation.

Tous les connecteurs utilisés doivent être conformes à la norme ISO 5356-1:1996; L'équipement anesthésique et respiratoire - Les connecteurs coniques - Partie 11 : Les cônes et les raccords et les circuits respiratoires avec la norme ISO 5367:2000; Les tubes raccords prévus pour utilisation avec les appareils et les ventilateurs d'anesthésie. Ces normes donnent les dimensions, les exigences fonctionnelles et les méthodes pour tester la force requise pour séparer les connecteurs mâles et femelles de 15 et $22 \mathrm{~mm}$. Les normes, qui doivent être maintenues après le nettoyage, spécifient que "les tubes raccords ne peuvent être séparés d'un connecteur mâle conique approprié par une force de moins de 40 Newtons"8 (un poids d'environ $4 \mathrm{~kg}$ suspendu aux tubulures). La pièce en Y ne peut être séparée du tube raccord par moins de 45 Newtons. ${ }^{9}$ Ces spécifications doivent être vérifiées après lavage.

L'achat de moniteurs, de circuits respiratoires et de connecteurs de qualité, le nettoyage convenable des articles réutilisables, ${ }^{10}$ la vérification attentive de l'usure de l'équipement avant l'usage, l'utilisation maximale de la technologie du monitorage, comprenant l'emploi des réglages appropriés des avertisseurs, et la vigilance face à l'état d'éveil réactionnel, tout cela peut contribuer aux meilleurs résultats possibles pour le patient.

\section{References}

I Kennedy RR, French RA. A breathing circuit disconnection detected by anesthetic agent monitoring. Can J Anesth 2001; 48: 847-49.

2 United States Food and Drug Administration Website on Reuse of Single Use Devices.

http://www.fda.gov/cdrh/reuse/index.shtml

3 Operator's Manual Document 889381-1 DatexOhmeda Helsinki Finland, June 1996.

4 International Electrotechnical Commission (IEC) and Organisation for International Standardization (ISO). IEC 60601-1-8. Medical electrical equipment part 1-8: general requirements for safety -6 . collateral standard: general requirements and guidelines for the application of alarms in medical electrical equipment. (Not yet published, target date November 2002). 
5 The Association for the Advancement of Medical

Instrumentation ANSI/AAMI. HE48:1993 - Human factors engineering guidelines and preferred practices for the design of medical devices.

http://www.aami.org

6 International Electrotechnical Commission (IEC). IEC 60601-1-6 Medical electrical equipment part 1: general requirements for safety, 6 . collateral standard: usability, analysis, test and validation of human factors compatibility. Comment document.

7 United States Food and Drug Administration Website on Human Factors http://www.fda.gov/cdrh/human factors.html

8 ISO 5367:2000; Breathing tubes intended for use with anaesthetic apparatus and ventilators. Organisation for International Standardization (ISO), 2000.

9 Anaesthetic and respiratory equipment - Conical connectors - Part 1: Cones and sockets. Organisation for International Standardization (ISO), ISO 5356-1, 1996.

10 Reprocessing of reusable medical and surgical supplies. CSA-International Z3148.8 - M88-CAN/CSA 